\section{mRCC: Alternatives Dosisregime gesucht}

\begin{abstract}
Das Standard-Dosisregime für Sunitinib zur initialen Therapie des metastasierten Nierenzellkarzinoms (mRCC) besteht aus $50 \mathrm{mg}$ täglich über 4 Wochen und 2-wöchiger Pause vor erneutem Beginn der Einnahme. Die Balance zwischen Toxizität und klinischem Nutzen ist allerdings eine Herausforderung.
\end{abstract}

$\mathrm{D}$ eshalb wurde in einer Phase-II-Studie jetzt ein intermittierendes Dosierungsschema nach initialer Standardtherapie mit Sunitinib bei 37 therapienaiven Patienten mit einem klarzelligen mRCC geprüft. Die Patienten erhielten zunächst 4 Zyklen mit 4 Wochen Sunitinib (50 mg pro Tag) und 2 Wochen Pause. Anschließend erfolgte eine Einschätzung des Ansprechens. Bei einer $\geq$ 10\%igen Reduktion der Tumorlast gegenüber dem Ausgangsbefund wurde die Sunitinib-Therapie unterbrochen, die Tumorlast alle 8 Wochen kontrolliert. Bei einem Anstieg über diesen Wert wurde wieder mit der Sunitinib-Behandlung begonnen. War im Restaging nach 4 initialen Zyklen Sunitinib keine Tumorlastverminderung um mindestens $10 \%$ festzustellen, erhielten die Pa- tienten 2 weitere Zyklen in Standarddosierung. War dann die Tumorlast entsprechend gesunken, konnte auch hier die Sunitinib-Therapie unterbrochen werden - bis zum erneuten Anstieg der Tumorlast. Die intermittierende Behandlung wurde fortgesetzt bis zum Progress nach RECIST oder bis zum Auftreten nicht tolerabler Toxizität.

Nach 4 Zyklen konnten 20 Patienten in die intermittierende Phase eintreten. Von den übrigen hatten 13 einen Progress entwickelt, einer nicht tolerierbare Nebenwirkungen und 3 Patienten widerriefen ihre Zustimmung zur Studienteilnahme vor Ende des 4. Zyklus.

Die objektive Ansprechrate bei den 20 verbliebenen Patienten lag nach $4 \mathrm{Zy}$ klen bei $46 \%$. In der Therapiepause nahm die Tumorlast gegenüber der Situ- ation vor Medikationsstopp im Median um $1,6 \mathrm{~cm}$ zu, die Spanne reichte von einer Abnahme um 2,9 cm bis zu einer $\mathrm{Zu}-$ nahme um 3,4 cm. Die meisten Patienten wiesen stabile sägezahnartige Muster einer Tumorlast-Abnahme bei Sunitinib-Einnahme und Zunahme bei Sunitinib-Pause auf. Aktuell liegt das mediane progressionsfreie Überleben der intermittiert behandelten Patienten bei 22,4 Monaten, das Gesamtüberleben bei 34,8 Monaten.

Fazit: Die Daten der Phase-II-Studie zeigen, dass eine intermittierende Strategie der Sunitinib-Therapie bei mRCC nach Ansprechen (Pause bei einer mindestens 10\%igen Reduktion der Tumorlast) machbar ist und eine anhaltende Kontrolle über die Erkrankung ermöglicht. In einer europäischen Phase-II/III-Studie wird diese Therapiestrategie derzeit mit der Standardbehandlung mit Sunitinib verglichen.

Friederike Klein

Ornstein MC et al. A Phase II Study of Intermittent Sunitinib in Previously Untreated Patients With Metastatic Renal Cell Carcinoma. J Clin Oncol. 2017;35(16):1764-9.

\title{
Weniger ist mehr bei mRCC
}

\section{Eine italienische Studiengruppe analysierte retrospektiv die Auswirkungen einer Dosisreduktion der Erstlinientherapie mit Sunitinib oder Pazopanib bei Patienten mit metastasiertem Nierenzellkarzinom (mRCC) .}

\footnotetext{
A usgewertet wurden die Daten von 591 an 11 Zentren in Italien behandelten Patienten, die wegen eines mRCC eine Erstlinientherapie mit Sunitinib (76,5\%) oder Pazopanib $(23,5 \%)$ in der Standarddosis erhalten hatten. Sunitinib wurde in der Dosis von $50 \mathrm{mg}$ täglich über 4 Wochen mit 2 Wochen Pause gegeben, Pazopanib in der Dosis von $800 \mathrm{mg}$ täglich durchgehend. Bei 45,7\% der Patienten musste wegen Toxizitäten die Dosis nach median 3,6 Monaten Behandlungszeit reduziert werden. Bei $44,5 \%$ der mit Sunitinib behandelten Patienten wurde die Dosis auf 37,5 mg verringert, bei 3,3\% auf $25 \mathrm{mg}$. Bei Pazopanib wurde die Dosis bei $33,1 \%$ der Patienten auf $600 \mathrm{mg}$ und bei $14,4 \%$ auf $400 \mathrm{mg}$ täglich reduziert. Die häufigsten Gründe für eine Do-
}

sisreduktion waren Asthenie (16,3\%), Hand-Fuß-Syndrom (13,3\%), Diarrhö (13,0\%) und Thrombozytopenie $(10,4 \%)$. Die Reduktion der Dosierung beeinträchtigte das Überleben nicht. Das nicht adjustierte progressionsfreie Überleben (PFS) lag in der Gruppe mit der Standarddosierung bei median 9,2 Monaten, bei reduzierter Dosis bei 18,1 Monaten (Hazard Ratio [HR] 1,74, 95\%-Konfidenzintervall [95\%-KI] 1,43-2,12; p < 0,001). Ein möglicher Vorteil der reduzierten Dosis spiegelte sich auch im Gesamtüberleben (OS) wider, das median 24,0 Monate unter Standarddosis und 49,4 Monate unter reduzierter Dosis betrug (HR 1,80, 95\%-KI 1,42-2,29; p < 0,001). Ein ähnliches Ergebnis für PFS und OS fand sich auch in den Subgruppen von
Patienten mit Sunitinib-Therapie alleine und von nephrektomierten Patienten. $45 \%$ der Patienten erhielten eine Zweitlinientherapie $(42,5 \%$ einen mTOR-Inhibitor und $54,1 \%$ einen Tyrosinkinaseinhibitor). Auch in dieser Situation zeigte sich noch ein Vorteil für Patienten mit reduzierter Dosis in der vorangegangenen Primärtherapie (OS: 19,8 Monate vs. 11,8 Monate bei Standarddosis; $\mathrm{p}=0,007$ ).

Fazit: Die häufig wegen Nebenwirkungen notwendige Dosisreduktion von Sunitinib oder Pazopanib in der Erstlinientherapie des mRCC ist prognostisch eher günstig. Die Forscher vermuten, dass das Auftreten dieser Nebenwirkungen einen Tumor- oder Patiententyp anzeigt, der besonders sensitiv auf die Therapie reagiert und deshalb gute Behandlungserfolge auch mit einer geringeren Dosis erreicht werden. Friederike Klein

lacovelli R et al. Clinical outcome of patients who reduced sunitinib or pazopanib during first-line treatment for advanced kidney cancer. Urol Oncol. 2017. May 29 [Epub ahead of print]. 